\title{
Gastroenteritis por Salmonella spp. en tres lactantes asociada a contacto con tortugas acuáticas
}

\author{
Stephanie Braun, Wanda Spalloni, Francisca Ferreccio, Javiera Postigo, Alda Fernández, \\ Lorena Porte, Alejandra Saldivia, Wilson Wigant y Vjera Triantafilo
}

\begin{abstract}
Hospital Militar de Santiago
Chile.

Laboratorio Clínico. Departamento de Pediatría, Infectología (SB, WS $L P, A S, W W, V T)$

Instituto de Salud Pública (AF) Universidad de Los Andes.

Programa de Internado (FF, JP) Clínica Alemana de Santiago.

Laboratorio Clínico (LP)

Los autores declaran no tener conflicto de interés para la publicación de este artículo.

Sin financiamiento.

Recibido: 10 de septiembre de 2014 Aceptado: 29 de enero de 2015
\end{abstract}

Correspondencia a: Stephanie Braun Jones stbraunj@gmail.com

\section{Introducción}

S almonella spp. es un bacilo gramnegativo que puede infectar a una gran variedad de hospederos, siendo responsable de aproximadamente 1,4 millones de casos de infección y 400 muertes al año en E.U.A ${ }^{1}$. En humanos, las infecciones por Salmonella no tífica se manifiestan generalmente como una gastroenteritis autolimitada. Sin embargo, en pacientes con factores de riesgo como niños bajo cinco años, inmunocomprometidos y adultos mayores, estas infecciones pueden producir meningitis, sepsis e incluso la muerte ${ }^{2,3}$.

En Chile, durante el período de vigilancia 2008-2011, el Instituto de Salud Pública (ISP) confirmó 11.403 aislados provenientes de muestras clínicas, con una tasa de incidencia de 21 por 100.000 habitantes en 2011. Se destaca que la mayor frecuencia de cepas proviene del grupo etario de 0 a 4 años $(19,4 \%)$, seguidos del grupo de 5 a 9 años $(14,9 \%)^{4}$.

Según la información disponible sobre enfermedades transmitidas por alimentos (ETA) del MINSAL al primer semestre de 2014, sólo $8 \%$ de los brotes tuvo agentes identificados y, de estos, Salmonella spp. correspondió a $88 \%{ }^{5}$.

Las infecciones por Salmonella spp. son causadas por ingesta de agua o alimentos contaminados, transmisión entre personas y exposición al ambiente o animales ${ }^{6}$. Los alimentos frecuentemente involucrados son huevos, lácteos y carne de ave $\mathrm{e}^{1,7}$. Los reservorios principales de Salmonella spp. son animales portadores asintomáticos. Los reptiles, referidos como "mascotas exóticas", son una fuente a menudo subestimada de salmonelosis ${ }^{8,9}$.
Los reptiles portan Salmonella spp. como parte de su microbiota intestinal, eliminando el microorganismo de forma intermitente a través de sus heces. Esta bacteria sobrevive en el ambiente y puede ser aislada desde superficies contaminadas por períodos prolongados ${ }^{9-11}$.

Aproximadamente $6 \%$ de las salmonelosis en E.U.A, son adquiridas por contacto directo o indirecto con reptiles 9 . Desde la década de los 50 en Europa y 60 en E.U.A, se han reportado casos de infecciones humanas asociadas a tortugas adquiridas como mascotas ${ }^{3,12,13}$. La especie más frecuentemente asociada a estas infecciones es Trachemys scripta elegans, conocida popularmente como tortuga acuática de orejas rojas ${ }^{3}$. El aumento de casos llevó al gobierno estadounidense a establecer políticas de control, intentando certificar tortugas libres de Salmonella spp. Sin embargo, esta medida no fue efectiva ${ }^{3,14}$. Finalmente, en 1975 la FDA prohibió la venta de tortugas pequeñas, (caparazón menor a $10 \mathrm{~cm}$ ), pero no su exportación. Se estimó que las tortugas con caparazones mayores a 10 $\mathrm{cm}$ no constituían un riesgo para los niños pequeños, pues serían muy grandes como para jugar con ellas. Se estima que esta medida evitó aproximadamente 100.000 casos de salmonelosis en niños por año ${ }^{3,15}$. No obstante, estos animales continúan siendo mascotas muy populares, reportándose en 2007 y 2008, un brote multiestatal de 100 casos de infecciones por Salmonella Paratyphi B var. Java, relacionado a tortugas pequeñas ${ }^{16}$.

La colonización de los reptiles con Salmonella spp. en tiendas de mascotas fue demostrado en estudios realizados en Brasil y Japón, con cifras de 39\% y $74 \%$, respectivamente ${ }^{17,18}$. En Colombia, se detectó presencia de Salmonella spp. en $33 \%$ de los estanques de Crocodylus 
intermedius y testudines cautivos y en $35 \%$ de tortugas en un centro de protección de vida silvestre ${ }^{19,20}$. Algunos serovares tales como Salmonella Abony, Newport, Paratyphi var Java, Litchfeld, Urbana, Stanley, Pomona y Poona entre otros han sido descritos en este tipo de infecciones ${ }^{2,3,13,16,21}$.

En Chile, la venta de tortugas no está regulada y pueden adquirirse tanto en el comercio formal como informal. En Valdivia, 13\% de muestras cloacales de 23 tortugas procedentes de tiendas de mascotas fueron positivas para Salmonella spp. ${ }^{22}$. En $70 \%$ de las muestras tomadas aleatoriamente durante 11 años (1997-2008) a reptiles ingresados a Chile a través de la Estación Cuarentenaria Pecuaria del Servicio Agrícola y Ganadero (SAG), se detectó la presencia de 15 serotipos de Salmonella spp., correspondiendo 13\% (n: 3) al serotipo Montevideo ${ }^{23}$. Un caso curioso descrito en Chile relata que un perro devoró unas tortugas y posteriormente tuvo diarrea. La dueña de las mascotas, de 2 años 5 meses, presentó diarrea por Salmonella Poona ${ }^{24}$.

Dada la alta importancia epidemiológica de este tema y la edad de los niños comprometidos, consideramos importante dar a conocer tres casos clínicos de salmonelosis asociados a tortugas. A nuestro entender, estos serían los primeros casos chilenos en los cuales ha sido posible confirmar a la tortuga como la fuente de infección.

\section{Caso clínico 1}

Lactante de cuatro meses, de sexo femenino, consultó en el Servicio de Urgencia Pediátrico (SUP) del Hospital Militar de Santiago (HMS) por presentar deposiciones líquidas con sangre de un día de evolución. La familia refirió que la mascota familiar era una tortuga acuática cuyo acuario se aseaba en el lavaplatos. No hubo otros miembros de la familia afectados.

$\mathrm{Al}$ momento de su ingreso, tenía un hemograma con 11.900 leucocitos $/ \mathrm{mm}^{3} ; 41,6 \%$ neutrófilos, VHS $32 \mathrm{~mm} / \mathrm{h}$ y PCR 50,4 mg/L. La ecografía abdominal fue normal. Los leucocitos fecales fueron positivos y los hemocultivos, negativos. Hubo desarrollo de Salmonella grupo C1 en el coprocultivo. La cepa fue enviada al ISP según norma ministerial vigente.

\section{Caso clínico 2}

Lactante de 1 año 3 meses de edad, sexo masculino, internado por vómitos, deshidratación, fiebre $39,5^{\circ} \mathrm{C}$ y diarrea. El hemograma mostró 7.700 leucocitos $/ \mathrm{mm}^{3}$ con $60,1 \%$ neutrófilos y $8 \%$ baciliformes; VHS $10 \mathrm{~mm} / \mathrm{h}$ y PCR 28,1 mg/L. En el coprocultivo hubo desarrollo de Salmonella grupo $\mathrm{C} 2$.

El padre y un hermano presentaron una diarrea autolimitada, por lo cual inicialmente se sospechó de un brote de intoxicación alimentaria. La familia había adquirido dos tortugas de orejas rojas en una feria libre y aseaba el acuario en un lavamanos de uso común. Además, el lactante jugaba con el reptil y con el agua del acuario. Se realizó coprocultivo a ambas tortugas.

\section{Caso clínico 3}

Lactante de 11 meses, de sexo femenino, fue internada por diarrea disentérica, fiebre y vómitos desde el día anterior. Presentaba hemograma con 2.700 leucocitos/ $\mathrm{mm}^{3}$ y PCR 190,9 mg/L. Evolucionó febril y con acidosis metabólica. Se inició tratamiento con cefotaxima, la que se mantuvo por dos días hasta su traslado a la UCI de otro centro asistencial. Fue dado de alta a los 6 días. Hubo desarrollo de Salmonella spp. en el coprocultivo. Ambos padres, una hermana y la asesora del hogar presentaron diarrea. Dos meses previos al ingreso, habían adquirido dos tortugas acuáticas en una feria libre. Se realizó coprocultivo a ambos reptiles. El aseo del acuario se realizaba en el lavaplatos, mismo lugar donde se preparaban los alimentos del lactante.

\section{Estudio microbiológico}

Se tomaron muestras de deposición a los pacientes y a las mascotas. La tórula de madera se introdujo en medio de transporte Cary Blair (BBL) para su traslado. Las muestras se sembraron en placas de agar Mac Conkey y agar SS (Bio-Mérieux) incubándose a $35^{\circ} \mathrm{C}$ en atmósfera ambiental. Se estudiaron las colonias sospechosas mediante pruebas bioquímicas tradicionales y tarjeta de identificación VITEK-ID gramnegativo (Biomérieux). Se realizó estudio de susceptibilidad a todos los aislados de Salmonella mediante difusión en disco según documento M100 S24 CLSI 2014. El estudio de serogrupo se realizó mediante reacción de aglutinación con antisuero polivalente (Salmonella O: poly A-I \& Vi, BD-Difco) y con antisueros específicos de grupo. Los aislados fueron enviados al Laboratorio de Referencia Nacional (ISP) para su serotipificación y subtipificación. Esta última fue realizada mediante electroforesis de campo pulsado (EFCP) utilizando la enzima de restricción XbaI, de acuerdo al protocolo estandarizado de la red PulseNet Latino América, OPS-CDC Atlanta (XXIX) ${ }^{25}$. Los patrones fueron analizados a través del programa bioinformático Bionumerics (Applyed Maths, EEUU.).

\section{Resultados microbiológicos}

Hubo desarrollo de Salmonella spp. en las deposiciones de los tres lactantes y en las deposiciones de dos de las cuatro tortugas estudiadas. Las mascotas infectadas correspondieron a una tortuga del caso 2 y a una del caso 3. 


\begin{tabular}{|llll|}
\multicolumn{5}{c}{} & \multicolumn{3}{c}{ Tabla 1. Características de los casos y sus mascotas } \\
\hline Característica & Caso 1 & Caso 2 & Caso 3 \\
Edad & 4 meses & 1 año 3 meses & 11 meses \\
Familiares afectados & No & Sí & Sí \\
Coprocultivo paciente & Salmonella Montevideo & Salmonella Newport & Salmonella Pomona \\
Coprocultivo tortuga & No & Sí & Sí \\
Nexo genético & No aplica & Salmonella Newport & Salmonella Pomona \\
\hline
\end{tabular}

\begin{tabular}{|l|l|ll|}
\hline & Subtipo PFGE & Aislamiento \\
CL-NP-XBA-014 & Caso Clínico 2 \\
CL-NP-XBA-014 & Mascota Tortuga \\
\end{tabular}

Figura 1. Electroforesis de campo pulsado, enzima de restricción Xbal. La subtipificación molecular muestra $100 \%$ de similitud en las dos cepas estudiadas.

En el primer caso, la cepa del paciente aglutinó con BD-Difco Salmonella O antisuero grupo C1 Factores 6,7. En el segundo caso, los aislados del paciente y de la tortuga aglutinaron con BD-Difco Salmonella $\mathrm{O}$ antisuero grupo C2 Factores 6,8. En el tercer caso, no fue posible sero-agrupar, ya que los aislados sólo aglutinaron con el antisuero polivalente.

La cepa del primer paciente fue identificada como Salmonella Montevideo; los aislados del segundo niño y su tortuga correspondieron a Salmonella Newport y los del tercer paciente y su tortuga, a Salmonella Pomona.

Todas las cepas fueron sensibles a ampicilina, cotrimoxazol, cloranfenicol y a ciprofloxacina excepto en el segundo caso, en que la cepa mostró susceptibilidad intermedia a esta quinolona.

Los resultados del estudio genético revelaron que los dos aislados del caso 2 presentaban un subtipo idéntico denominado CL-NP-XBA-014 (100\% de similitud) (Figura 1). Por su parte, ambos aislados del caso 3 presentaban el subtipo $\mathrm{Cl}-\mathrm{Spm}-\mathrm{Xba}$-001. Estos hallazgos confirmaron el nexo genético entre los casos y las mascotas.

\section{Discusión}

La salmonelosis asociada a reptiles constituye un problema de salud pública no resuelto, siendo la zoonosis más comúnmente relacionada a este tipo de animales. Sin embargo, aún existe desconocimiento de los padres con respecto al riesgo potencial de infección al que se exponen ellos y sus familias. Las posibles vías de transmisión son las manos contaminadas con deposiciones de las mascotas y, de forma indirecta, a través de la preparación de los alimentos en lavaplatos o cocinas contaminadas con agua de los acuarios ${ }^{3,11}$. Por esto, uno de las medidas de prevención fundamentales es el lavado de manos ${ }^{26}$.

En dos de los casos que describimos, la infección afectó al lactante y a otros miembros del grupo familiar. Por lo tanto, es importante consultar siempre sobre el antecedente de la tenencia de mascotas, especialmente reptiles, en el estudio de brotes de gastroenteritis. También es fundamental tomar muestras, no solo de los pacientes sino de las mascotas supuestamente involucradas. No obstante, debe tenerse en cuenta que el rendimiento del coprocultivo puede verse afectado por eliminación intermitente de Salmonella en reptiles y por el muestreo único de heces y no de la cloaca u otros sitios $3,8,11,27$.

Varios autores han descrito que los niños, en especial los más pequeños, son los más afectados ${ }^{2,6,27,28}$. Este hecho concuerda con lo observado en nuestra serie. En Inglaterra, Murphy comparó los casos de salmonelosis asociados a reptiles con los no asociados, en niños bajo los 5 años. El $27 \%$ del total se asoció a reptiles y presentó edades significativamente menores a la del grupo no asociado a reptiles ( 0,5 vs 1 año). Además, este grupo tenía 2,5 veces mayor probabilidad de hospitalización ${ }^{28}$. Los serotipos encontrados habían sido descritos previamente en infecciones relacionadas a reptiles; Newport y Pomona a tortugas y Montevideo a serpientes ${ }^{3,6,11,27}$.

Salmonella Newport ha sido aislada en productos cárnicos, ganado y tomates, y corresponde a $11,4 \%$ de Salmonella spp. reportadas por el CDC en $2011^{30,33}$. En Chile ha sido aislada en lobos marinos ${ }^{34}$. Salmonella Montevideo constituye 2,6\% de Salmonella spp. reportadas por el CDC en 2011 y, en nuestro país, se encontró en 13\% de las muestras de reptiles estudiadas por el $\mathrm{SAG}^{23,33}$. Salmonella Pomona ha sido recuperada de lactantes en Europa, E.U.A. y China ${ }^{6,21,27,35}$. En E.U.A. se comunicó la muerte de un lactante de 3 semanas de vida en quien se aisló este serotipo en sangre y $\mathrm{LCR}^{21}$. Un estudio chino encontró Salmonella Pomona en 39\% de las tortugas de orejas rojas exóticas en una reserva en Guangdon, considerándola como altamente patogénica ${ }^{35}$. La evolución más grave de la paciente de nuestro tercer caso clínico concuerda con esa publicación.

El uso de herramientas moleculares estandarizadas como la EFCP representa un gran apoyo en la investigación de los casos, en especial frente a patógenos como Salmonella enterica, ya que permiten de forma certera la identificación de la fuente responsable de infección ${ }^{25}$.

El gran número de reptiles importados (82.016 especímenes de E.U.A. entre 2003 y 2005), la alta tasa de portación de Salmonella spp. y el desconocimiento general acerca del riesgo relacionado a mascotas exóticas, urge la implementación de medidas epidemiológicas para el control de esta zoonosis ${ }^{23}$. La educación de la población, el control de la venta de reptiles y la alerta a la comunidad 
médica son algunos de las medidas necesarias para evitar infecciones, especialmente en lactantes.

\section{Resumen}

Las infecciones por Salmonella no tíficas, son una importante causa de diarrea aguda en niños. Generalmente adquiridas por alimentos contaminados, el contacto con animales, especialmente reptiles, también es responsable de esta zoonosis. Describimos tres casos de lactantes con gastroenteritis por Salmonella ( $S$. Montevideo, $S$. Newport y $S$. Pomona), relacionados a contacto con tortugas mascotas. En dos de los casos se aisló Salmonella en las deposiciones de los niños y de las tortugas. En todos hubo proximidad entre los niños y los acuarios y/o tortugas, siendo probable la contaminación en la preparación de alimentos. El estudio de clonalidad por electroforesis de campo pulsado demostró el mismo subtipo genético en el aislado correspondiente al paciente y en el procedente de la mascota, lo cual confirmó la fuente zoonótica de los casos. Esta zoonosis debe ser abordada por las autoridades de salud y las sociedades científicas, ya que representa un importante riesgo para la salud, especialmente de niños pequeños.

\section{Referencias bibliográficas}

1.- Voetsch A C, Van Gilder T J, Angulo F J, Farley M M, Shallow S, Marcus R, et al. Food Net estimate of the burden of illness caused by nontyphoidal Salmonella infections in the United States. Clin Infect Dis 2004; 38 Suppl 3: S127-34.

2.- Van Meervene E, Botteldorn N, Lokietek S, Vatlet M, Cupa A, Naranjo M, et al. Turtle-associated Salmonella septicaemia and meningitis in a 2-month-old baby. J Med Microbiol 2009; 58: 1379-81.

3.- Harris J R, Neil K P, Behravesh C B, Sotir M J, Angulo F J. Recent multistate outbreaks of human Salmonella infections acquired from turtles: a continuing public health challenge. Clin Infect Dis 2010; 50: 554-9.

4.- Instituto de Salud Pública. Vigilancia de Salmonella spp Chile, 2008-2011. Boletin ISP, 2012; 13: (2) 1-15.

5.- Informe (ETA) Enfermedades Entéricas Semana 40, 2014. Disponible. http://epi.minsal. $\mathrm{cl} / \mathrm{epi} / \mathrm{html} /$ bolets/reportes/Entericas/Informe Entericas_SE402014.pdf\#page=4, 2014 . (Consultado el 17 de octubre de 2014).

6.- Bertrand S, Rimhanen-Finne R, Weill F X, Rabsch W, Thornton L, Perevoscikovs J, et al. Salmonella infections associated with reptiles: the current situation in Europe. Euro Surveill 2008; 13 (24): pii18902.

7.- Alexandre M, Pozo C, González V, Martínez M, Prat S, Fernández A, et al. Detección de Salmonella Enteritidis en muestras de productos avícolas de consumo humano en la Región Metropolitana. Rev Med Chile 2000; 128: 1075-83.

8.- Warwick C, Lambiris A J, Westwood D, Steedman C. Reptile related salmonellosis. J R Soc Med 2001; 94: 124-6.

9.- Mermin J, Hutwagner L, Vugia D, Shallow S, Daily P, Bender J, et al. Reptiles, amphibians and human Salmonella infection: a population-based, case-control study.
Clin Infect Dis 2004; 38 Suppl 3: S253-61.

10.- Warwick C, Arena P C, Steedman C. Visitor behaviour and public health implications associated with exotic pet markets: an observational study. J R S M Short Rep 2012; 3: 63.

11.- Chiodini R J, Sundberg J P. Salmonellosis in reptiles: a review. Am J Epidemiol 1981; 113 : 494-9.

12.- Williams L P Jr, Helsdon H L. Pet turtles as a cause of human salmonellosis. JAMA 1965; 192: 347-51.

13.- Lamm S H, Taylor A Jr, Gangarosa E J, Anderson H W, Young W, Clark M H, et al. Turtle-associated salmonelosis, I: an estimation of the magnitude of the problem in the United States, 1970-1971. Am J Epidemiol 1972; 95: 511-7.

14.- Polk L D. Salmonellosis in children from pet turtles certified Salmonella free. Clin Pediatr 1974; 13: 719-20.

15.- Cohen M L, Potter M, Pollard R, Feldman R A. Turtle-associated salmonellosis in the United States: effect of public health action, 1970 to 1976. JAMA 1980; 243: 1247-9.

16.- CDC. Multistate outbreak of human Salmonella infections associated with exposure to turtlesUnited States, 2007-2008. MMWR Morb Mortal Wkly Rep 2008; 57: 69-72.

17.- Abalem de Sá I V, Solari C A. Salmonella in brazilian and imported pet reptiles. Braz J Microbiol 2001; 32: 293-7.

18.- Nakadai A, Kuroki T, Kato Y, Suzuki R, Yamai S, Yaginuma C, et al. Prevalence of Salmonella spp. in pet reptiles in Japan. J Vet Med Sci, 2005; 67: 97-101.

19.- Pachón D, Pulido A, Moreno C. Aislamiento y serotipificación de Salmonella sp. en estanques con Crocodylus intermedius y testudines cautivos en Villavicencio-Colombia. Rev MVZ 2011; 16: 2564-75.

20.- Sánchez-Jiménez M M, Rincón-Ruiz P A, Duque S, Giraldo M A, Ramírez-Monroy D M, Jaramillo G, et al. Salmonella enterica in semi- aquatic turtles in Colombia. J Infect Dev Ctries 2011; 5: 361-4.

21.- CDC. Turtle-Associated salmonellosis in humans-United States, 2006-2007. MMWR Morb Mortal Wkly Rep 2007; 56: 649-52.

22.- Toledo F. Detección de Salmonella spp, en tortugas de orejas rojas (Trachemys scripta elegans) en la ciudad de Valdivia. 2009; Memoria de título, Instituto de Medicina Preventiva Veterinaria, Facultad de Ciencias Veterinarias, Universidad Austral de Chile.

23.- Osorio M, Saldías M E, Valiente K. Estudio retrospectivo de presencia de Salmonella spp. en reptiles ingresados a Chile a través de la Estación Cuarentenaria Pecuaria, 1997-2008. Boletín Veterinario Oficial, BVO, $\mathrm{N}^{\circ} 12$, Servicio Agrícola y Ganadero. Santiago, Chile. 10 pp. Disponible en: <http://www2.sag.gob.cl/Pecuaria/bvo/ BVO_12_II_semestre_2010/PDF_articulos/ estudio_salmonella_lab.pdf. (Consultado en agosto de 2014).

24.- Vargas G. Zoonosis en el hogar. Rev Chilena Infectol 2013; 30: 689.

25.- Ribot E M, Fair M A, Gautom R, Cameron D N, Hunter S B, Swaminathan B, et al. Standardization of pulsed-field gel electrophoresis protocols for the subtyping of Escherichia coli O157:H7, Salmonella, and Shigella for PulseNet. F. Foodborne Pathog Dis 2006; 3: 9-67.

26.- Friedman C R, Torigian C, Shillam P J, Hoffman R E, Heltzel D, Beebe J L, et al. An outbreak of salmonellosis among children attending a reptile exhibit at a zoo. J Pediatr 1998; 132: 802-7.

27.- Pees M, Rabsch W, Plenz B, Fruth A, Prager R, Simon S, et al. Evidence for the transmission of Salmonella from reptiles to children in Germany, July 2010 to October 2011. Euro Surveill 2013; 18: pii: 20634.

28.- Murphy D, Oshin F. Reptile-associated salmonellosis in children aged under 5 years 
in South West England. Arch Dis Child 2015; 100: 364-5.

29.- Fonseca R J, Dubey L M. Salmonella Montevideo sepsis from a pet snake. Pediatr Infect Dis J 1994; 13: 550.

30.- Foley S L, Lynne A M, Nayak R. Salmonella challenges: prevalence in swine and poultry and potential pathogenicity of such isolates. J Anim Sci 2008; 86 (14 Suppl): E149-62.

31.- Clark S. Salmonella Newport-An emerging disease in dairy cattle. Indiana Animal Disease Diagnostic Laboratory, Summer 2004 Newsletter. http://www.addl.purdue. edu/newsletters/2004/summer/salmnewp.htm. (Consultado el 20 de julio de 2014).

32.- Greene S K, Daly E R, Talbot E A, Demma L J, Holzbauer S, Patel N J, et al. Recurrent multistate outbreak of Salmonella Newport associated with tomatoes from contaminated fields, 2005. Epidemiol Infect 2008; 136: 157-65.

33.- CDC. http://www.cdc.gov/nationalsurveillance/ PDFs/NationalSalmSurveillOverview_508.

pdf. [En línea] 2011. (Consultado en agosto de 2014).

34.- Sturm N, Abalos P, Fernández A, Rodríguez G,
Oviedo P, Arroyo V, et al. Salmonella enterica in pinnipeds, Chile [letter]. Emerg Infect Dis 2011; 17: 2377-8

35.- Gong S, Wang F, Shi H, Zhou P, Ge Y, Hua L, et al. Highly pathogenic Salmonella Pomona was first isolated from the exotic red-eared slider (Trachemys scripta elegans) in the wild in China: Implications for public health. Sci Total Environ 2014; 468-469: 28-30.

37.- Seepersadsingh N, Adesiyun A. Prevalence and antimicrobial resistance of Salmonella spp. in pet mammals, reptiles, fish aquarium water, and birds in Trinidad. J Vet Med 2003; 50: 488-93. 